\title{
EDITORIAL
}

\section{The second pandemic: a call to action}

Pediatric Research (2022) 91:2-3; https://doi.org/10.1038/s41390021-01461-2

We are at risk for a second pandemic but not one of coronavirus disease 2019 (COVID-19). We are at risk for a wave of easily preventable infections due to the dramatic decline in vaccine rates across the country. Researchers at the Centers for Disease Control and Prevention analyzed data from the federal Vaccines for Children Program and the Vaccine Safety Datalink. They compared pre- and post-COVID vaccine administration and showed 3 million fewer vaccines were given between 2019 and 2020 during the period of January-April. ${ }^{1}$ In Michigan, more than half the children became behind in their vaccines due to the pandemic. ${ }^{2}$ The Blue Cross Blue Shield Association, which insures $>100$ million lives, has reported that childhood vaccine rates have declined $26 \%$ in 2020 compared to 2019 , threatening herd immunity. ${ }^{3}$ World-wide vaccine rates are down 50\%, which have already caused dangerous outbreaks of measles and polio. ${ }^{4}$ In the United States, despite recent social distancing precautions, measles has reached an all-time high of 1282 cases across 31 states, up from 55 from as recently as $2012 .^{5}$ A generation of medical students may have never seen the pox from the varicella virus. Given that vaccine rates have dramatically decreased, there is a good chance that they will now. Without a thoughtful plan, we face worse outbreaks of easily preventable illnesses.

The decline in vaccine rates has been driven by a sensible response due to the pandemic. State regulations placed restrictions on travel outside of the home. The message from the press was clear: stay safe, self-isolate. Anecdotal stories of outbreaks in schools, offices, and social gatherings affirmed this message. Families appropriately responded. They stayed home, deferring routine care until the cloud of the pandemic had lifted. Many practices quickly adapted to a telehealth platform to enable continued care and patient connection.

As we emerge from the pandemic, we face an important challenge. How do we vaccinate our children against easily preventable infections? In the same way that we quickly mobilized telehealth platforms and office safety protocols, now is the time to mobilize a quick response to vaccinate our children. While the rollout of the COVID-19 vaccine is critical, we must also pay attention to a second wave of easily preventable infections. This second pandemic may bring with it more than just communicable disease. Vaccination delays serve as a clear and quantifiable alarm for a broader loss of preventative care. At these same visits, growth is monitored, development is assessed, and screens for vision, hearing, and lead are performed.

This will require a new way to practice medicine: panel management. We must identify those patients who are behind in their vaccines and then actively reach out to them for administration. Every practice needs to employ a population health approach to their patient population. We must leverage our electronic medical records (EMRs) for the power that lies within. We suggest a call to action.

To make this a reality, several things must occur. First, physicians need to commit. Our practices are organized to treat patients one by one. We are a piece-meal operation. It is time to treat our patients as a community. How many of our children are behind in their lead screening? Who has missed their Modified Checklist for Autism in Toddlers? If a child does not appear for a routine visit, we miss them and they fall behind in their vaccines and routine screening. We need to do a better job of proactive outreach. Some will say that this places yet more administrative burdens on physicians, but if we fail to act, we risk significant illness for our patients. In an era where we are increasingly paid based on quality indicators, we need to make this a priority. If we will not lead this effort, who will?

Second, our Electronic Health Records (EHRs) need to make these reports easier to generate. We can now order a pair of glasses, a plane ticket to Paris, or a signed Mickey Mantle baseball card within minutes. Why is it so complicated to generate a report on vaccine rates on our patient panels? Our EHRs are geared toward documentation and billing, with templated visits and pull-forward documentation. Our EHRs are powerful repositories of accurate, current data. We need to leverage this data to take better care of our patients. EHRs should make this possible with a few keystrokes. Commercial enterprises (think Google and Amazon) make this intuitive and easy. EPIC, Cerner, and the rest should take a cue from these companies and streamline these processes.

Third, institutions must allocate resources; and insurance companies must pay for these services. Historically, insurance companies have occasionally faxed notifications about care coordination attempts and who is behind in vaccine administration. While this may be a good faith attempt, such sporadic notices fall outside the work flow of routine primary care, are disconnected from our EMRs, and often are out of date. Since most practices now belong to larger institutions, we need to leverage institutional resources to identify and reach out to these patients. While physician leadership is critical, we believe it is most appropriate and efficient to operationalize this among nursing leaders and clinic managers. These tasks should not be in addition to an already busy job jar. Hiring nursing leaders and outreach workers to organize these projects, while embedded in the patient's medical home, is more effective. Critics of these projects will appropriately point to the increased expense and infrastructure. However, similar projects have shown improved compliance with medication adherence and vaccine administration in under-resourced settings in the developing world. ${ }^{6}$ We should be at least as efficient as a Sleeping Sickness eradication program in Malawi or a River Blindness program in Ecuador. As we have much greater resources, it is embarrassing that we do not have similar programs.

The COVID pandemic has disproportionately affected ethnic minorities and those who live in impoverished socio-economic settings. ${ }^{7}$ This second pandemic threatens to similarly impact those most vulnerable in our communities, making this work more urgent. This frame-shift in primary care has been a long time coming. Barriers to innovation have been the inertia of our entrenched patterns of care-the CPT code approach to physician reimbursement, the already significant administrative burdens by nurses and physicians alike. The upside of improved panel management shows great promise. Our patients will receive better care and easily preventable infections will diminish. We hope the sequella of this pandemic will be a renewed approach to the communities we serve. 


\section{ADDITIONAL INFORMATION}

Competing interests: The authors declare no competing interests.

Publisher's note Springer Nature remains neutral with regard to jurisdictional claims in published maps and institutional affiliations.

Benjamin R. Doolittle (iD) and Andrew Loza ${ }^{2}$ ${ }^{1}$ Department of Internal Medicine \& Pediatrics, Yale University School of Medicine, New Haven, CT, USA and ${ }^{2}$ Combined Internal Medicine and Pediatrics Program, Yale-New Haven Hospital, New Haven, CT, USA Correspondence: Benjamin R. Doolittle (Benjamin.doolittle@yale.edu)

\section{REFERENCES}

1. Santoli, J. M. et al. Effects of the COVID-19 pandemic on routine pediatric vaccine ordering and administration — United States, 2020. MMWR Morb. Mortal. Wkly Rep. 69, 591-593 (2020).
2. Bramer, C. A. et al. Decline in child vaccination coverage during the COVID-19 pandemic - Michigan Care Improvement Registry, May 2016-May 2020. MMWR Morb. Mortal. Wkly Rep. 69, 630-631 (2020).

3. Blue Cross Blue Shield. Missing vaccinations during COVID-19 puts our children \& communities at risk. https://www.bcbs.com/the-health-of-america/infographics/ missing-vaccinations-during-covid-19-puts-our-children-and-communities-at-risk (2020).

4. World Health Organization. Emergency call to action. https://s3.amazonaws.com/ wp-agility2/measles/wp-content/uploads/2020/11/Call-To-Action-20201105.pdf (2020).

5. Centers for Disease Control and Prevention. Measles cases and outbreaks. https://www.cdc.gov/measles/cases-outbreaks.html (2021).

6. Scott, K. et al. What do we know about community-based health worker programs? A systematic review of existing reviews on community health workers. Hum. Resour. Health 16, 1-7 (2018).

7. Peek, M. E. et al. COVID-19 among African Americans: an action plan for mitigating disparities. Am. J. Public Health 111, 286-292 (2021). 